\title{
Gene looping between the viral LTRs is a hallmark of transcriptionally active HIV-I and Mo-MLV proviral DNA Chiara Vardabasso ${ }^{* 1}$, Marina Lusic ${ }^{1}$, Nick Proudfoot $^{2}$ and Mauro Giacca ${ }^{1}$
}

Address: ${ }^{1}$ Molecular Medicine Laboratory, International Centre for Genetic Engineering and Biotechnology (ICGEB), Trieste Italy and ${ }^{2}$ Sir William Dunn School of Pathology, University of Oxford, UK

* Corresponding author

from Frontiers of Retrovirology: Complex retroviruses, retroelements and their hosts Montpellier, France. 21-23 September 2009

Published: 24 September 2009

Retrovirology 2009, 6(Suppl 2):P90 doi:I0.1186/1742-4690-6-S2-P90

This abstract is available from: http://www.retrovirology.com/content/6/S2/P90

(c) 2009 Vardabasso et al; licensee BioMed Central Ltd.

By exploiting the Chromosome Conformation Capture (3C) technique, we have previously shown that the HIV-1 provirus forms a transcription-dependent gene loop structure between the 5' LTR promoter and 3' LTR poly(A) signal. Flavopiridol-mediated inhibition of RNA polymerase II elongation blocked 5' to 3' LTR juxtaposition, indicating that this structure is maintained during transcription [1].

The formation of such high order chromatin structure, if aberrantly generated between a retroviral LTR sequence and the promoter of a neighboring gene, has the potential to activate transcription of the cellular gene. This would provide a molecular explanation to the insertional mutagenesis events observed in several gene therapy clinical trials entailing the use of retroviral vectors, leading to the activation of cellular protoconcogenes by means of longrange chromosomal interactions. Indeed, despite the vast amount of data on the insertion sites of retroviral vectors in preclinical and clinical studies, the molecular mechanisms connecting retrovirus integration to aberrant cellular gene activation are still almost completely obscure.

We first wanted to test whether LTR-mediated gene looping is also a characteristics of Mo-MLV-based retroviral vectors. In this respect, compared to HIV-1, Mo-MLV has a much weaker poly(A) signal, having thus higher potential to mobilize cellular sequences due to the frequent $3^{\prime}$ poly(A) read-through. We produced and characterized a number of HeLa clones harboring single integrations of a Mo-MLV vector carrying a selectable gene. Using the 3C technique, we observed that, in all the analyzed clones, a gene loop was present juxtaposing the 5 ' and 3' LTRs of the vector. Thus, gene looping is not a prerogative of HIV1 but a characteristic also extended to oncoretroviruses.

We are currently in the process of analyzing, in our clones, the levels of expression of the cellular genes neighboring the proviral integration sites within regions spanning 50 $\mathrm{kb}$ upstream or downstream of the insertions and correlating the eventual changes with the formation of aberrant loops. In addition, 3C experiments are also performed in a series of HaCaT clones in which up-regulation of genes surrounding retroviral integration has already been demonstrated (in collaboration with F. Mavilio, Milan, Italy) and will later be extended to cell clones from patients with SCID-X1 who were enrolled in one of the recent gene therapy clinical trials (with A. Thrasher, London, UK).

\section{References}

I. Perkins KJ, et al.: Transcription-dependent gene looping of the HIV-I provirus is dictated by recognition of pre-mRNA processing signals. Mol Cell 2008, 29:56-68. 